\title{
DPC VS TDMA IN SISO BROADCAST CHANNELS: A PRACTICAL COMPARISON
}

\author{
G.R. Mohammad-Khani *, S. Lasaulce ${ }^{1}$, J. Dumont ${ }^{2}$ \\ ${ }^{1}$ LSS CNRS, 3 rue Joliot-Curie, 91190 Gif-sur-Yvette, France, \{khani,lasaulce $\} @ 1$ lss.supelec.fr \\ ${ }^{2}$ France Telecom R\&D, rue du Général Leclerc, 92794 Issy-les-Moulineaux, France, julien.dumont@ francetelecom.com
}

\begin{abstract}
In this paper we want to make a fair comparison between TDMA and DPC in terms of the BER region and maximum number of satisfied users. For this purpose we choose the coding/decoding schemes as follows : DPC is based on a trellis coded quantization implementation and successive coding while TDMA uses trellis coded modulation. Simulation results are provided both for the perfect and imperfect channel state information cases. In the perfect CSI case all the tested scenarios show that TDMA generally outperfoms DPC. In the imperfect CSI case DPC is shown to be less sensitive than TDMA to channel estimation errors.
\end{abstract}

Keywords -Broadcast channel, TDMA, Dirty paper, TCQ, Nested lattices, TCM

\section{INTRODUCTION}

Consider the situation where one single-antenna transmitter sends independent messages to several single-antenna receivers. This situation is commonly referred to as the single input single output (SISO) broadcast channel (BC). The capacity region of the coherent Gaussian BC (GBC) is well known and has shown to strictly enlarge the TDMA rate region [1]. The GBC capacity region can be achieved by using dirty paper coding (DPC), which has been proposed by [2] for the AWG "noise plus interference" channel $Y=X+Z+S$. For this channel the interference part $(S)$ is assumed to be non-causally known to the transmitter only. Under this assumption Costa showed that DPC achieves the same maximum rate as if the interference were not present (or equivalently if the receiver knew the interference term perfectly). This result has been generalized to Gaussian multiple input multiple output (MIMO) BCs [3].

Taken into account the information-theoretic optimality of DPC some authors have quantified the loss induced by using TDMA instead of DPC in Gaussian broadcast channels. For example, in [4] the authors studied the ratio of the maximal sum rate provided by DPC to that provided by TDMA as a function of the number of transmit antennas (say $t$ ), receive antennas (say $r$ ), active users and the transmit power. This ratio is always greater than 1 but can be very close to 1 in certain situations (e.g. for small transmit powers and/or for small $t / r$ ratios). On the other hand, in [5], Yang and Belfiore considered situations where using DPC instead of TDMA leads to significant performance losses in terms of (achievable) data rate region. For a 2-user SISO Gaussian BC the authors studied the impact of imperfect channel knowledge at the transmitter on 353 the DPC rate region. From their simulation results the authors concluded that DPC is very sensitive to channel estimation errors and thefore the DPC rate region does no longer include that of TDMA. Their comparison between TDMA and DPC is in fact not totally fair since imperfect CSIT (CSI at the Tx) is assumed while perfect CSI is assumed at the receivers. The TDMA rate region is then not affected by their assumptions (only CSIR - CSI at the Rx - matters in TDMA).

In this paper we tackle the problem of the BC versus TDMA comparison from a different perspective. Whereas [4] and [5] made a comparison in terms of maximum achievable data rates, we focus here on the performance in terms of bit error rates (BER). For this purpose we can no longer use information theoretic DPC but have to make a particular choice for implementing DPC. For DPC we use Trellis Coded Quantization (TCQ), initially used by [6] for data hiding. This trellisbased DPC approach has also been used by Yu et al. [7] for the single-user channel with Tx side information. In order to have comparable complexities, we use Trellis Coded Modulation (TCM, see [13]) for implementing the different channel coders in TDMA. In this setup we want to compare a multiple access scheme based on successive coding and DPC with TDMA in terms of BER regions and the maximum number of satisfied users. Additionally we want to assess the influence of imperfect CSI at both sides of the transmission on the performance of the considered multiple access schemes. For this purpose we first provide our signal model (section II.), then we describe the coding/decoding schemes under investigation (sections III. and IV.). The comparison in terms of BERs and numbers of satisfied users is made in section $\mathrm{V}$. for different spectral efficiencies, channel asymmetry degrees and channel knowledge degrees. Section VI. summarizes the main conclusions and observations from the present work.

\section{SIGNAL MODEL}

For each user $k \in\{1, \ldots, K\}$ the received signal writes as :

$$
Y_{k}=h_{k} X+Z_{k}
$$

where $X \in \mathbb{C}$ is the baseband transmitted signal, $h_{k}$ is the complex path gain from the transmitter to receiver $k, Z_{k} \sim$ $\mathcal{N}\left(0, N_{k} B\right)$ is the complex circular symmetric AWGN channel noise for user $k$ and $B$ is the useful signal bandwidth. In this paper the channel gains $\left\{h_{1}, \ldots, h_{K}\right\}$ are assumed to be static. The transmitted signal is subject to a power constraint $E\left[|X|^{2}\right] \leq \bar{P}$. Without loss of generality we assume that 
$\forall k \in\{1, \ldots K\}, E\left[\left|Z_{k}\right|^{2}\right]=N_{k} B=1$. Note also that we implicitly assumed ISI-free channels (no inter-symbol interference).

The information message intended for user $k$ is denoted by $W_{k} \in\left\{1, \ldots, 2^{n \bar{R}_{k}}\right\}$ where $n$ is the length of a transmitted data block and $\bar{R}_{k}$ is the information data rate in bit per channel use (bpcu). Then the information messages are encoded, which gives the transmit signal $X$. As we will see $X$ can be either in a finite alphabet (TDMA case) or a continuous one (DPC case).

\section{TRANSMITTER DESCRIPTION}

\section{A. DPC-based transmitter (figure 1)}

The inputs of the DPC-based transmitter are the information messages $W_{1}, \ldots, W_{K}$ and the channel gains $h_{1}, \ldots, h_{K}$. Like in a CDMA system the transmitter output $X$ is assumed to be the sum of $K$ signals : $X=\sum_{k} X_{k}$, which means in practice that these signals use the same frequency and time slots. Each signal $X_{k}$, intended for user $k$, has a power $\bar{P}_{k}=E\left[\left|X_{k}\right|^{2}\right]$ such that $\sum_{k} \bar{P}_{k} \leq \bar{P}$. Each signal $X_{k}$ is generated by a dirty paper encoder. This is what figure 1 shows : The transmitter comprises a bank of dirty paper encoders linked according to the successive coding idea [8]. Each DP encoder, say encoder $k$, is fed with the information message $W_{k}$ and the outputs previously encoded. Therefore the signal received by user $k$ can be rewritten as :

$$
Y_{k}=\underbrace{h_{k} X_{k}}_{X_{k}^{\prime}}+\underbrace{\sum_{j<k} h_{k} X_{j}}_{S_{k}}+\underbrace{\sum_{j>k} h_{k} X_{j}+Z_{k}}_{Z_{k}^{\prime}} .
$$

Each subchannel $Y_{k}=X_{k}^{\prime}+Z_{k}^{\prime}+S_{k}$ is a Costa's channel with :

$$
\begin{aligned}
& -E\left[\left|X_{k}^{\prime}\right|^{2}\right]=\left|h_{k}\right|^{2} \bar{P}_{k} \triangleq \bar{P}_{k}^{\prime} \\
& -E\left[\left|S_{k}\right|^{2}\right]=\sum_{j<k}\left|h_{k}\right|^{2} \bar{P}_{j} \triangleq \bar{Q}_{k} \\
& -E\left[\left|Z_{k}^{\prime}\right|^{2}\right]=\sum_{j>k}\left|h_{k}\right|^{2} \bar{P}_{j} \triangleq N_{k}^{\prime} B .
\end{aligned}
$$

Then for each user $k$ the multiuser interference $\left(S_{k}\right)$ associated with the users previously encoded can be cancelled out. Note that a priori the different information messages can be encoded in any order. In fact, the best information-theoretic order (leading to the capacity region) is to encode the users from the lowest to the highest channel signal-to-noise ratio ( $S N R_{k} \triangleq\left|h_{k}\right|^{2}$ in our setup). However, if we are interested in a different performance criterion such as the maximum number of satisfied users the optimum order can be different. In this paper a user is said to be satisfied if, for a given data rate $\bar{R}_{k}$, the target BER is reached i.e. $P_{e k} \leq P_{e k}^{(0)}$. The same kind of comments can be made regarding the power allocation issue. It is known that the SISO GBC sum rate $\sum_{k} \bar{R}_{k}$ is maximized by allocating the whole power to the user having the best SNR. Obviously a different power allocation scheme has to be used for satisfying the maximum number of users e.g. when the best user only needs a low data rate with a low quality of service.

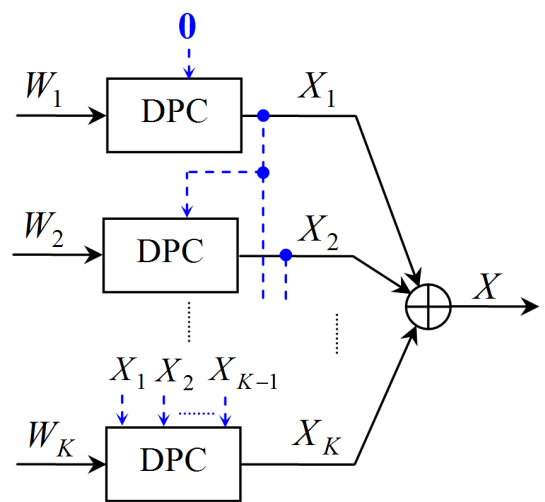

FIG. 1 - Transmitter structure for DPC

\section{B. Dirty paper encoder description}

We have seen that the DPC-based transmitter is made of a bank of $K$ DP encoders. In this section we describe the structure of the DP encoder itself. To better understand how it works we briefly review the corresponding theoretic coding scheme [2]. Costa showed that a 2-stage encoder structure is optimal in terms of data rate. The outer stage generates from the information message $W_{k}$ and $S_{k}$ an auxiliary signal, called $U_{k}$. The inner stage linearly combines $S_{k}$ and $U_{k}$ as follows : $X_{k}^{\prime}=U_{k}-\alpha_{k} S_{k}$ where $U_{k}$ is constructed such that ${ }^{1}$ $I\left(X_{k} ; S_{k}\right)=0$. The optimum $\alpha_{k}$, in the sense of $\operatorname{Costa}^{2}$, is merely an MMSE weighting : $\alpha_{k}^{*}=P_{k}^{\prime} /\left(P_{k}^{\prime}+N_{k}^{\prime} B\right)$. By a way of comparison, the corresponding Tomlinson Harashima scheme (THS) would use a ZF weighting i.e. $\alpha_{k}=1$.

Assuming $^{3}$ that we want to mimic Costa's coder structure in practice, the main point is then to know how to generate the auxiliary signal $U_{k}$. The most efficient DP strategy would be to use general nested lattices (NL) [10], [11]. However the construction of such lattices for reasonably high dimensions is not systematic and the underlying coding/decoding complexity can be very high. On the other hand if the complexity issue matters the most, one can use the THS or scalar Costa's scheme (SCS) introduced by Eggers et al. in [9]. In this paper we chose to implement DPC through a trellis coded quantization, which implements in fact a suboptimum nested lattice. The TCQ has at least the two advantages that it allows for a relatively simple construction of an equivalent nested lattice and it offers a good trade-off between performance and complexity. A nested lattice comprises two lattices : A coarse and a fine lattice. The coarse lattice (say $\Lambda_{2}$ ) implements a good source code (i.e. the quantizer or modulo-operator used to implement Costa's scheme) while the fine lattice (say $\Lambda_{1}$ ) implements a good channel code. So the TCQ can be seen as a NL in which $\Lambda_{2}$ is fixed by the choice of the quantizer used on each

${ }^{1} \mathrm{I}(\mathrm{X} ; \mathrm{S})$ denotes the mutual information between $\mathrm{X}$ and $\mathrm{S}$

${ }^{2}$ To be precise the corresponding $\alpha_{k}$ maximizes $I\left(U_{k} ; Y_{k}\right)-I\left(U_{k} ; S_{k}\right)$.

${ }^{3}$ We have to mention that mimicing information theoretic DP structure has been shown to be fruitful for the Costa's channel, even in terms of BERs as shown by [9] 
transition of the trellis and the trellis structure allows us to generate a coset code $\left(\Lambda_{1}\right)$. For example, if one chooses a scalar quantizer, the equivalent coarse lattice is cubic.

Now we use the nested lattices interpretation to describe how the TCQ-based DP encoder works. For each user "k", the signal $\underline{X}_{k}^{\prime}$ is constructed as follows :

$$
\underline{X}_{k}^{\prime}=\underline{C}_{k}-\alpha_{k} \underline{S}_{k}+\underline{D}_{k} \bmod \Delta \mathbb{Z}^{n} .
$$

Let us comment this construction. The sequence of $n \times R_{k}$ information bits for user " $k$ " is mapped, through the trellis, into a sequence of "n" cosets. For each information message $W_{k}$ the coset is denoted by $\underline{C}_{k}$. The coset $\underline{C}_{k}$ comprises all the points of $\Lambda_{1}$ that are located in the fundamental Voronoï cell of $\Lambda_{2}$. Note that the coset implements precisely the idea of the auxiliary variable that we mentioned previously : Indeed the auxiliary signal $\underline{U}_{k}$ is implicitly generated by the DP encoder who selects the codeword that is as close as possible (modulo operator) to the signal $\alpha_{k} \underline{S}_{k}$. The signal $\underline{D}_{k}$ is a dithering signal known both to the encoder and decoder. It insures the independence between $\underline{X}_{k}^{\prime}$ and $\underline{S}_{k}$. Also because of dithering $\underline{X}_{k}^{\prime}$ has a uniform distribution on the Voronoï region $\Lambda_{2}$. The power constraint can then be easily adjusted, through the parameter $\Delta$, by choosing an appropriate size for the Voronoï region. For a scalar quantizer $\left(\Lambda_{2}\right.$ is cubic) $: E\left[|X|^{2}\right]=\Delta^{2} / 6$.

In practice the TCQ is implemented like a TCM [13]. The $R_{k}=\frac{1}{n} \log _{2}\left|\mathcal{W}_{k}\right|$ bits associated with user $k$ are split into two parts $\left(\mathcal{W}_{k}\right.$ is the message alphabet of user $\left.k\right)$. One part is encoded by a convolutional encoder and used for selecting a subset of quantizers and the other is used to select a quantizer within a given subset.

\section{TDMA-based transmitter (figure 2)}

In the TDMA transmitter each information message $W_{k}$ is encoded separately. In order to make a fair comparison with DPC, each encoder generates the signal $X_{k}$ by using a trellis coded modulation (comparable complexities) and the same spectral efficiencies (same data rates). In each frame, the $K$ TCM are then selected according to the TDMA scheme. Let $T_{k}$ be the time duration in which user $k$ is selected. Then the average data rate and transmit power for user $k$ are given by

$$
\bar{R}_{k}=\frac{T_{k}}{\sum_{k=1}^{K} T_{k}} R_{k}, \bar{P}_{k}=\frac{T_{k}}{\sum_{k=1}^{K} T_{k}} P_{k}
$$

where $R_{k}$ would represent the spectral efficiency for user $k$ if he were given all the time slots. Because of synchronization issues at the receivers, real TDMA systems assume equal time slot durations : $\forall k \in\{1, \ldots, K\}, T_{k}=T / K$ where $T$ is the frame duration. As a consequence, if user $k$ has a data rate $\bar{R}_{k}$ with DPC, TDMA has to use a modulation with a higher order i.e. $R_{k}=K \times \bar{R}_{k}$. At this point we can emphasize the main issue in the proposed comparison. The two advantages of TDMA over DPC is that there is no multiple access interference and the "instantaneous" power $P_{k}$ (over a given time slot) can vary from 0 to $K \times \bar{P}$. On the other hand, as the users transmits simultaneously in DPC, TDMA has to compensate the data rate loss induced by time division by increasing the modulation order so that the data rates remain comparable.

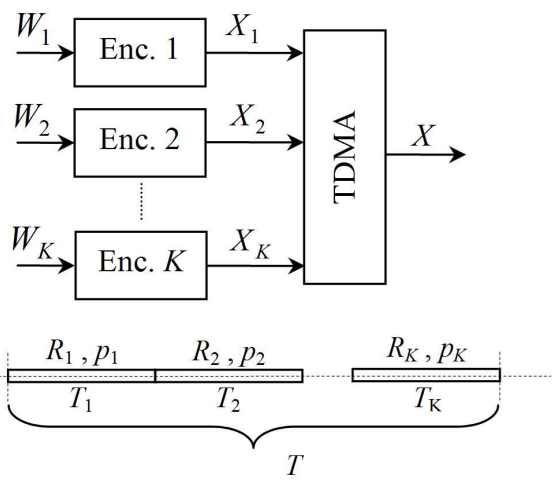

FIG. 2 - Transmitter structure for TDMA

\section{Channel KNOWLEDGE AT THE RECEIVERS}

\section{A. Channel state information assumptions}

We have seen that the DPC approach is based on the channel knowledge i.e. the path gains $h_{1}, \ldots, h_{K}$ at the transmitter. Although the "causal" multiuser interference has been cancelled out at the transmitter, the receiver still needs a certain knowledge on the channel. Each receiver " $k$ " decodes the information message as follows :

$$
\underline{\hat{C}}_{k}=\alpha_{k} \underline{Y}_{k}-\underline{D}_{k} \bmod \Delta \mathbb{Z}^{n} .
$$

This corresponds to finding the nearest coset with respect to the received signal $\underline{Y}_{k}$. More specifically the decoder implements a Viterbi algorithm for the search of the optimum sequence of cosets. To implement equation (5) and compute the branch metrics of the trellis receiver "k" needs to know its weight $\alpha_{k}=P_{k}^{\prime} /\left(P_{k}^{\prime}+N_{k}^{\prime} B\right)$. This amounts to know the individual SINR at each receiver. In a cellular system this assumption is reasonable. In our context (static channel) it is more restrictive because knowing $S I N R_{k}$ means knowing $\left|h_{k}\right|^{2}$. Therefore in the special case of Gaussian broadcast channels, implementing DPC requires channel state information both at the transmitter and the receivers. Of course if no CSI is available at the receivers, one can still use a ZF implementation of DPC (e.g. the Tomlinson Harashima scheme) since $\alpha_{k}=1$.

As for the TDMA transmitter, it does not need (a priori) any channel state information at the transmitter but it is mandatory at the receivers for coherent detection (in order to compute the branch metrics for the Viterbi algorithm used to decode the TCM).

\section{B. Channel estimation}

To take into account channel estimation errors we use a simple modeling for estimation errors. First, we assume that 
the channel estimates at the receivers comes from a trainingbased estimation procedure. Each receiver estimates its channel from an $m$-symbol training sequence. Second we assume that the channel estimation accuracy at the transmitter is similar to that of the receivers. For instance this condition is met in the following two cases : 1 . The transmitter has a noiseless feedback of the receive channel estimates. 2. The transmitter uses itself an $m$-symbol training sequence and assumes uplink-downlink channel reciprocity (e.g. in time division duplex systems). Assuming a training sequence with good correlation properties we can approximate the channel estimation error variance as follows (see e.g. [12]) :

$$
\forall k \in\{1, \ldots, K\}, E\left[\left|\hat{h}_{k}-h_{k}\right|^{2}\right]=N_{k} B \frac{\ell}{m}
$$

where $\ell \leq m$ is the channel impulse response length i.e. $\ell=1$ in our setup. Under these assumptions note that the ratio of the channel noise power to the channel estimation noise power is always less than 1 .

\section{DPC-TDMA COMPARISON : SIMULATION RESULTS}

\section{A. Simulation setup}

The situation under consideration is the same as in [5] : 2user SISO Gaussian broadcast channel, static channel gains. But here we make a fairer comparison between DPC and TDMA regarding CSI knowledge at the transmitter/receiver. Indeed [5] assumes perfect CSI at the receiver and imperfect CSI at the transmitter. This assumption gives an advantage to the TDMA scheme because only CSI at the receivers is needed. Here we assume estimation errors at both sides of the channels.

In order to draw conclusions that are not too much channeldependent we considered different scenarios. A scenario can be characterized by the target spectral efficiencies (in bpcu), the "broadcast channel asymmetry" and the estimation noise level. The BC asymmetry is merely characterized by the ratios : $\forall k \in\{2, \ldots, K\}, \gamma_{k}=S N R_{1} / S N R_{k}=\left|h_{1}\right|^{2} /\left|h_{k}\right|^{2}$. We always assume $K=2$. Considered scenarios :

- $\left(R_{1}, R_{2}\right) \in\{(1,1),(2,2),(2,1)\}$;

$-\gamma_{2} \in\{0 \mathrm{~dB}, 5 \mathrm{~dB}\}$;

$-\forall k \Leftrightarrow\{1,2\}, \rho_{k}^{(e s t)} \triangleq \quad \frac{N_{k} B}{E\left[\left|\hat{h}_{k}-h_{k}\right|^{2}\right]} \in$

$\{10 \mathrm{~dB}, 15 \mathrm{~dB}, 20 \mathrm{~dB}, 100 \mathrm{~dB}\}$ (the latter value is assumed to correspond to the perfect CSI case).

To achieve the rate $R_{1}=1 \mathrm{bpcu}$ with DPC we use a TCQ based on a 4-QAM constellation (coarse lattice, see figure 3-a) plus a $1 / 2$ 16-state convolutional encoder. For implementing TDMA with this rate, a TCM with an 8-QAM (figure 3-b) and the same convolutional encoder are used. To achieve the rate $R_{1}=2$ bpcu with DPC we use a TCQ based on a 8-QAM constellation (coarse lattice, see figure 3 -a) plus a $1 / 2$ 16-state convolutional encoder. For TDMA the 8-QAM is replaced with a 32-QAM (figure 3-b).

At last let us mention that the channels are chosen randomly and kept fixed for all the simulation. The transmit power $P$ is chosen such that realistic BER values are obtained.

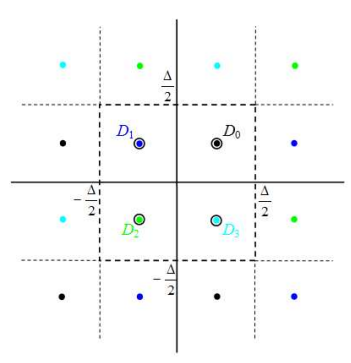

(a)
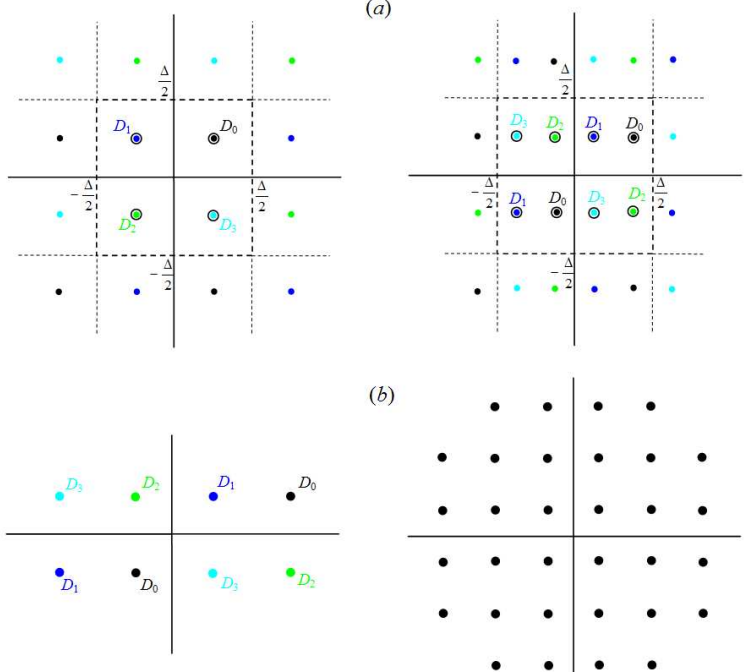

(b)

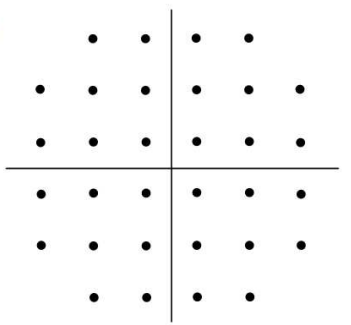

FIG. 3 - Coarse lattices for (a) TCQ - 4-QAM and TCQ - 8-QAM and constellations for (b) TCM - 8-QAM and TCQ - 32-QAM

\section{B. Simulation results}

First, assuming perfect CSI at the transmitter and receivers $(\rho=100 \mathrm{~dB})$, we compare the performance of DPC to that of TDMA for a symmetric BC : $\gamma_{2}=0 \mathrm{~dB}$. Figures 4 and 5 represent the achievable BER regions for the cases $R_{1}=R_{2}=1 \mathrm{bpcu}$ and $R_{1}=R_{2}=2 \mathrm{bpcu}$ respectively. To obtain these regions one just needs to vary the power allocation between the two users : $\bar{P}=\bar{P}_{1}+\bar{P}_{2}=\beta \bar{P}+(1-\beta) \bar{P}$ with $\beta \in[0,1]$. In both cases it appears that TDMA generally outperforms DPC, the difference is even more marked for higher rates. This is the contrary to what is known for (theoretical) achievable rate regions. Although this observation is made for a special choice for the coding schemes, it is still interesting since the TCM is known to be a good coding scheme for Gaussian channels and the TCQ is also a good DPC scheme (see e.g. [6]). By changing the BC asymmetry degree $\left(\gamma_{2}=5 \mathrm{~dB}\right)$ or coding rates quite similar conclusions are obtained (see figures 6,7). In fact we also observed the same tendency for other scenarios e.g. by increasing the number of users to $K=3$. This is what figures 10 and 11 illustrates in terms of number of satistified users : except in the low transmit power regime, TDMA performs better than successive coding. These simulations results mean that increasing the constellation size (for compensating the data rate loss due to time division) in a TCM-based TDMA system induces less performance degradation than the increase of multiple access interference with DPC. Indeed in a DPC system, assuming the users to be order according to their individual signal-to-noise ratio $\left(S N R_{1} \geq S N R_{2} \geq \ldots S N R_{K}\right)$, user $k$ sees k-1 interferers. It seems that this residual interference can play an important role in the overall system performance, as our simulations show. At last note that DPC performs better than TDMA (figure 4) when 
the power allocated to user 1 (resp. user 2) strongly dominates that of user 2 (resp. user 1).

Now we look at the impact of imperfect CSI on the performance of TDMA and DPC. For $R_{1}=R_{2}=1 \mathrm{bpcu}$ and $\gamma_{2}=0 \mathrm{~dB}$ figure 8 shows that TDMA is more sensitive to channel estimate errors than DPC. As for the perfect CSI case we see that DPC takes the advantage over TDMA when the power allocated to user 1 (resp. user 2) dominates that of user 2 (resp. user 1). We have found the same behavior for higher coding rates (figure 9). These simulations show that DPC is not as sensitive as expected [5]. Once again the behavior of the BER regions is the opposite to that forecasted by the theory for achievable rate regions [5].

\section{CONCLUSIONS}

In terms of coding rate region dirty paper coding is known to be a more efficient multiple access scheme than TDMA. Always in terms of achievable rates [5] suggests that DPC is very sensitive to channel estimation errors.

Based on a choice of coding schemes for implementing DPC (trellis coded quantization) and TDMA (using trellis coded modulation) we compared the bit error rate regions achieved by these schemes in different simulation scenarios (different coding rates, broadcast channel asymmety degrees, channel noise levels). Note that comparing TCQ with TCM is fair in terms of complexity and also makes sense since these schemes are known to be efficient for Gaussian channels. Our simulations results show that TDMA generally outperforms DPC except when the power allocated to user 1 (resp. user 2) dominates that of user 2 (resp. user 1). In the latter case DPC provides lower bit error rates. At last DPC is shown to be less sensitive to channel estimation errors than TDMA. All these results show that there exist practical coding schemes for which the DPC-TDMA comparison leads to conclusions that markedly differ from what is usually forecasted by information-theoretic analyses.

\section{REFERENCES}

[1] T. M. Cover and J.A. Thomas, "Elements of Information Theory", John Wiley, New York, 1990.

[2] M. H. M. Costa, "Writing on dirty paper", IEEE Trans. on Inform. Theory, IT-29(3), pp. 439-441, 1983

[3] H. Weingarten and Y. Steinberg, and S. Shamai, "The capacity region of the Gaussian MIMO broadcast channel”, CCIT Report No 491, Teknion Institute, Haifa, Israel, June 2004.

[4] N. Jindal, and A. Goldsmith, "Dirty-paper coding versus TDMA for MIMO Broadcast channels", IEEE Transactions on Information Theory, Volume 51, Issue 5, pp. 1783-1794, May 2005.

[5] S. Yang and J.C. Belfiore, "The Impact of Channel Estimation Error on the DPC Region of the Two-User Gaussian Broadcast Channel", in 43rd Allerton Conference, Monticello, IL, Sep. 2005.

[6] J. Chou and S. Pradhan and L. El Ghaoui, and K. Ramchandran, "A robust optimization solution to the data hiding problem using distributed source coding principles, in Proc. SPIE : Image Video Commun. Process, vol. 3974, Jan. 2000
[7] W. Yu, D. P. Varodayan and J. M. Cioffi, "Trellis and convolutional precoding for transmitter-based presubstraction", IEEE Trans. on Comm., COM53(7), pp. 1220-1230, July 2005.

[8] G. Caire and S. Shamai, "On the achievable throughput of a multiantenna Gaussian broadcast channel", IEEE Trans. Inform. Theory, IT-49(7), pp. 1691-1706, 2003.

[9] J. Eggers and R. Bäuml and R. Tzschoppe, and B. Girod, "Scalar Costa scheme for information embedding", IEEE Trans. on Signal Proc., SP51(4), pp. 1003-1019, 2003.

[10] R. Zamir and S. Shamai, and U. Erez, "Nested linear/lattice codes for structured multiterminal binning", IEEE Trans. on Inform. Theory, IT48(6), pp. 1250-1276, 2002.

[11] U. Erez and R. Zamir, "Achieving on the AWGN channel with lattice encoding and decoding", IEEE Trans. on Inform. Theory, IT-50(10), pp. 2293-2314, 2004.

[12] S. Lasaulce, and N. Sellami, "On the impact of using unreliable data on the bootstrap channel estimation performance", in Proc. Signal Processing for Advanced Wireless Communications (SPAWC)", June 2003.

[13] G. Ungerboeck, "Trellis-Coded Modulation with Redundant Signal Sets", in IEEE Comm. Mag., vol. 25, no. 2, pp. 5-21, 1987.

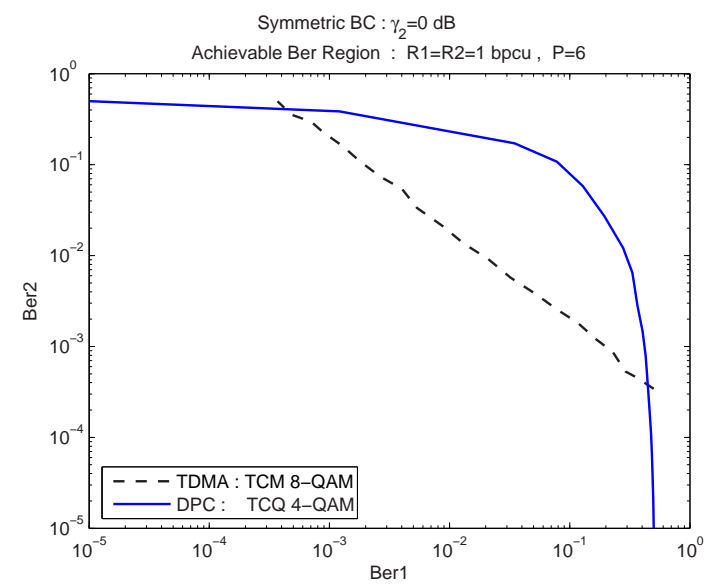

FIG. 4 - Achievable BER regions for DPC and TDMA for a symmetric BC $\left(\gamma_{2}=0 \mathrm{~dB}\right)$ and $R_{1}=R_{2}=1 \mathrm{bpcu}$

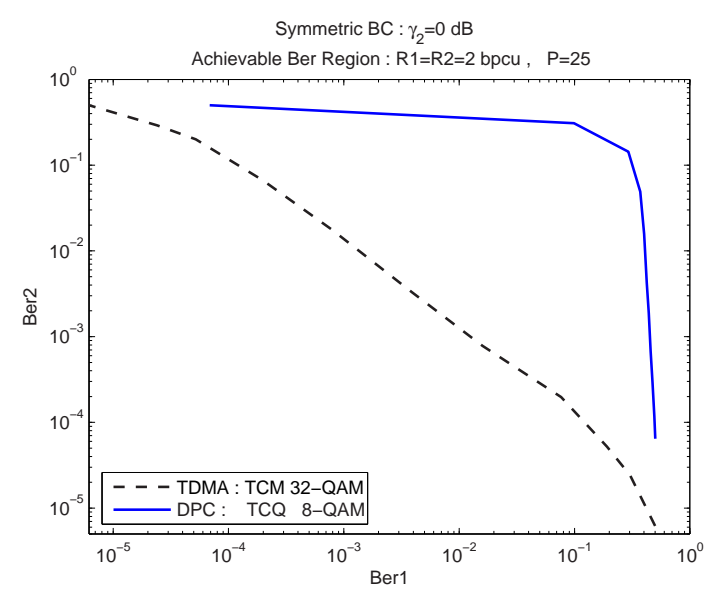

FIG. 5 - Achievable BER regions for DPC and TDMA for a symmetric BC $\left(\gamma_{2}=0 \mathrm{~dB}\right)$ and $R_{1}=R_{2}=2 \mathrm{bpcu}$ 


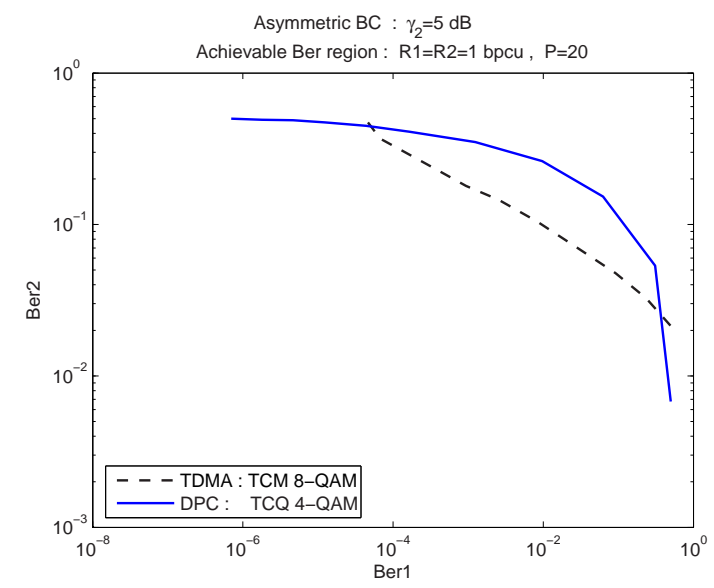

FIG. 6 - Achievable BER regions for DPC and TDMA for an asymmetric $\mathrm{BC}\left(\gamma_{2}=5 \mathrm{~dB}\right)$ and $R_{1}=R_{2}=2 \mathrm{bpcu}$

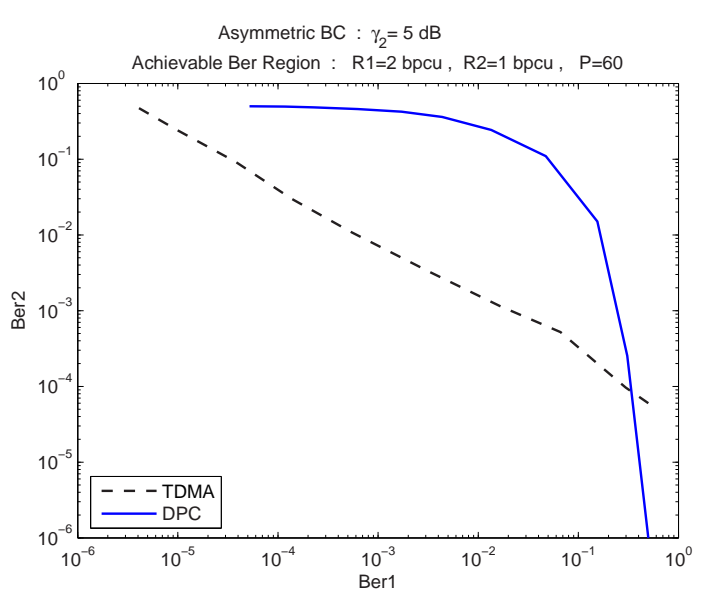

FIG. 7 - Achievable BER regions for DPC and TDMA for an asymmetric $\mathrm{BC}\left(\gamma_{2}=5 \mathrm{~dB}\right)$ and $R_{1}=2 \mathrm{bpcu}, R_{2}=1 \mathrm{bpcu}$

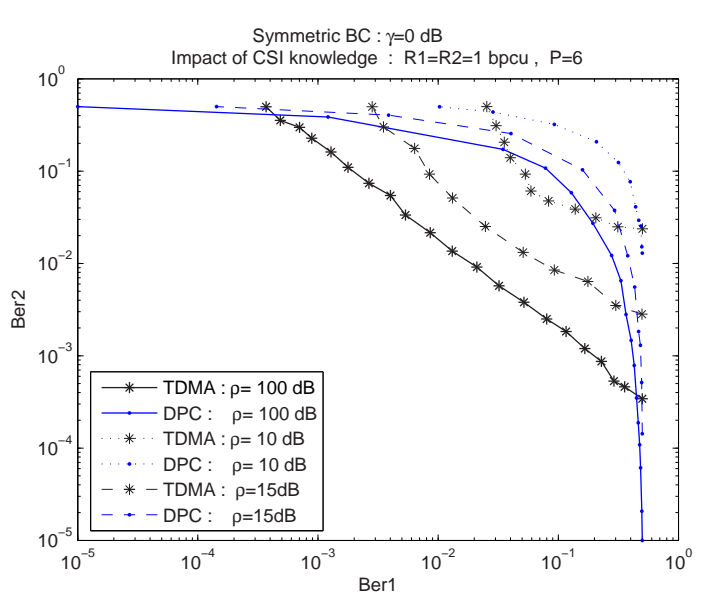

FIG. 8 - Impact of channel estimation errors for $R_{1}=R_{2}=1 \mathrm{bpcu}$

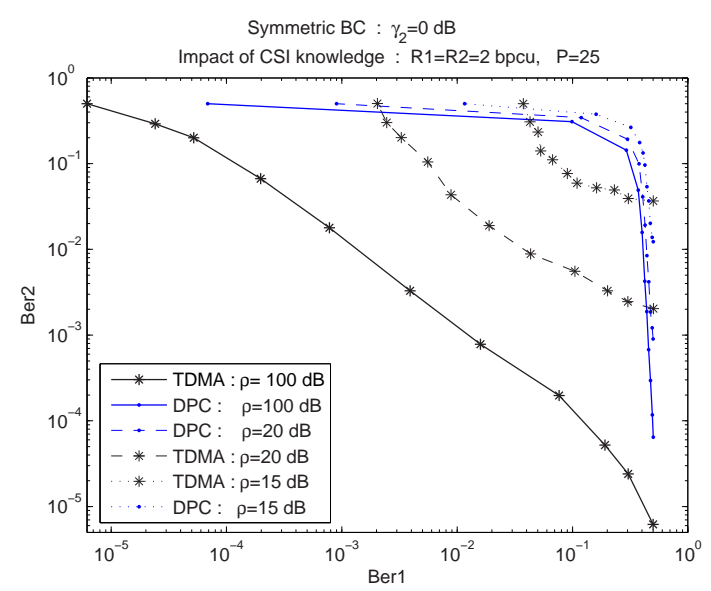

FIG. 9 - Impact of channel estimation errors for $R_{1}=R_{2}=2 \mathrm{bpcu}$

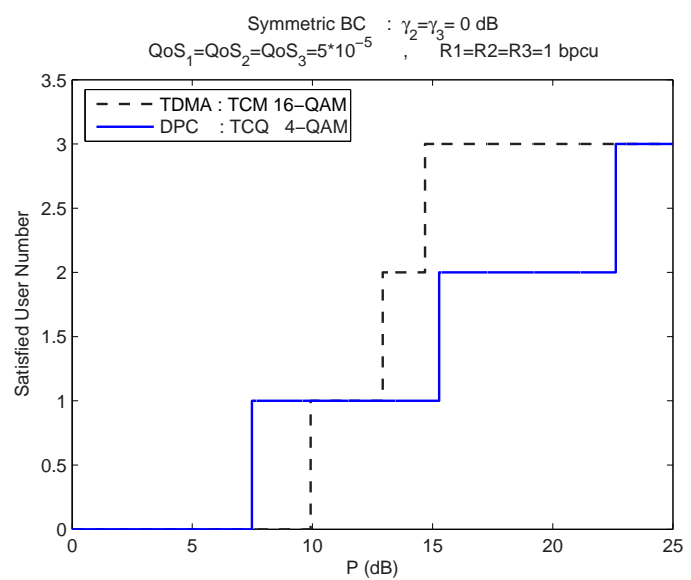

FIG. 10 - Number of satisfied users versus transmit power for $R_{1}=R_{2}=$ $1 \mathrm{bpcu}$

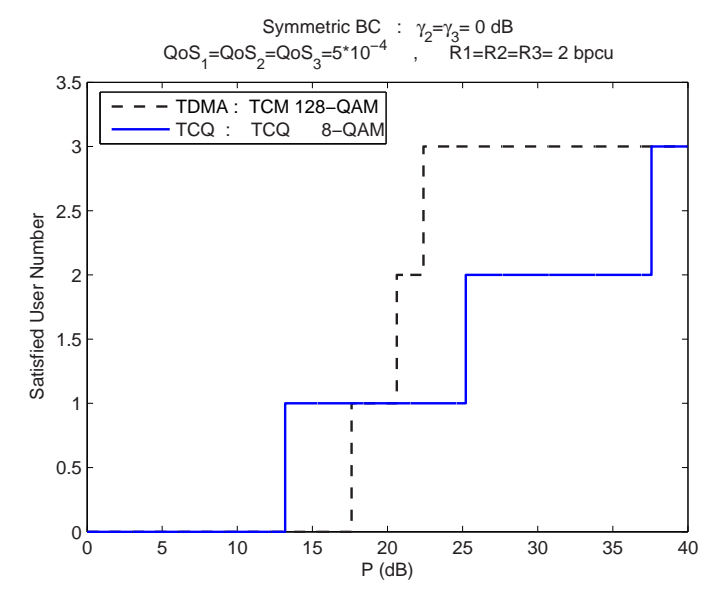

FIG. 11 - Number of satisfied users versus transmit power for $R_{1}=R_{2}=$ 2 bpcu 